\title{
Comparative analysis of performance characteristics of nozzle heads for powder transportation in a laser cladding and direct material deposition
}

\author{
Oleg Kovalev',* \\ ${ }^{1}$ Khristianovich Institute of Theoretical and Applied Mechanics SB RAS, Novosibirsk, Russia
}

\begin{abstract}
The analysis of the dynamics of gas-dispersed flows created by nozzle heads, which are used in the additive technology of direct laser deposition of material in the manufacture of metal products of complex geometry, is analyzed. The features of using nozzle systems for increasing the efficiency of powder transport in laser treatment are discussed. Typical gas-powder transport nozzles of well-known manufacturers Trumpf and Precitec are considered. The study is conducted to obtain additional information for a thorough understanding of the physical processes that take place and to increase the efficiency of the practical use of equipment.
\end{abstract}

\section{Introduction}

The methods of laser cladding and direct material deposition (DMD) are used not only to receive coatings and restore parts, but also to produce 3D items of complex spatial shape [1]. The deposition of a new layer on the creating item takes place in a laser-induced molten pool. The powder material added to the molten pool gets metallurgical connection to the substrate, and if necessary, the HAZ zone can be minimal (from hundreds of microns to millimeters). The supply of powder plays a key role in this process, having a decisive influence on the accuracy and size of the treatment area and the deposition of the material, as well as on the properties of the product and the efficiency of the use of the added powder. There are several techniques to organize the feeding of powder into the laser's operating area. For this purpose, there are specially designed nozzles $[1,2]$, Fig. 1, the choice of which depends on the technical requirements for the product and the properties of the powder materials used.

Based on the principles of lateral or coaxial powder supplying for laser cladding and direct depositing of materials, Trumpf, Precetec and Fraunhofer have developed special powder feeding systems. These systems can transport powder materials with particle sizes from 20 to $150 \mu \mathrm{m}$ for the depositing of macrolayers with a thickness of more than $100 \mu \mathrm{m}$, and with particle sizes less than $20 \mu \mathrm{m}$ for depositing microlayers with a thickness of less

\footnotetext{
* Corresponding author: kovalev@itam.nsc.ru
} 
than $100 \mu \mathrm{m}$. The nozzles are suitable for most lasers, which are currently used for laser deposition of $\mathrm{CO}_{2}, \mathrm{Nd}$ : YAG, disk, fiber and diode lasers.
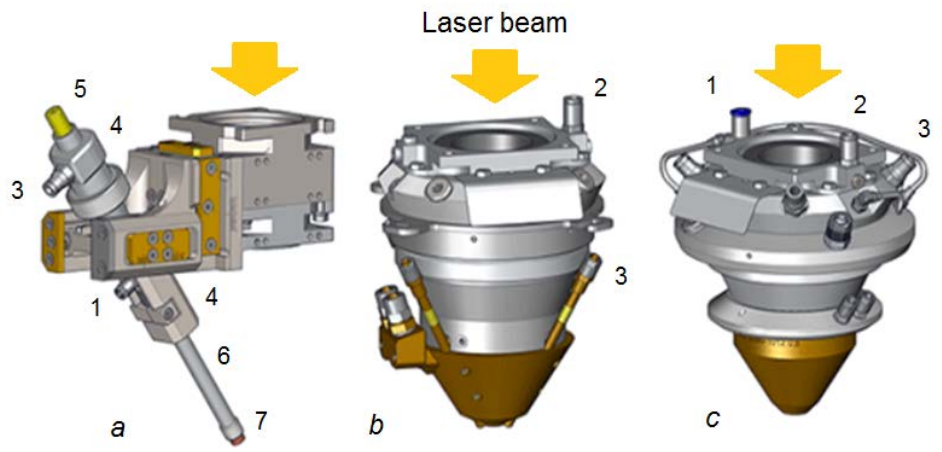

Fig. 1. Different types of Precitec nozzles for transporting powdered materials: lateral stream (a); four-stream (b); annular stream (c). The numbers indicate the input channels: 1-carrier gas; 2shielding gas; 3-powder; and the components: 4- cyclone; 5- cap; 6 - branch pipe; 7- injector.

The industrial application of laser cladding and direct depositing of powder items is connected with a number of technological factors: the characteristics of the laser beam, the thermal and physical properties of the original powder, the quality of the resulting items, which in turn depend on the operating parameters of the powder feeding systems, the focusing of gas-dispersed flows, the type of assisted gases and much more. For the development of rational modes of depositing materials and optimizing the industrial manufacturing, mathematical modeling and various types of diagnostics are used that can predict fast processes.

The development of rational modes only empirically is very laborious, and is associated with the costs of depreciation of expensive equipment used for these purposes and powder materials. The use of numerical and diagnostic methods allows predicting the processes and making adjustments to processing parameters, nozzle design, etc.

It was indicative that the gasdynamic processes in laser cladding should be calculated within the framework of viscous and necessarily compressible gas models, and it is necessary to include in the calculation not only the external flow between the nozzle and the substrate, as previously thought, but also the flows inside the channels of the nozzle [3].

Changes in the design of process equipment lead to changes in modeling methods, so it is difficult to automate calculations. This explains the absence at the present time of specialized and functionally oriented software for calculating the processes of laser interaction with materials during their processing.

\section{Motivation}

Equipment for 3D printing in Russia is not produced. Among specialists, there is such a practice when researchers try to create the nozzles for powder transportation in laser cladding independently, without giving importance to the requirements to which these nozzles should satisfy. There are a limited number of foreign manufacturers of such nozzles that comply with these requirements. The lack of a detailed description of the mechanisms of operation of imported equipment hinders its effective use by Russian consumers. Studies on the transport properties of nozzles to improve the technology of laser cladding in open printing are still not enough. In this paper, a comparative analysis of the design features of typical nozzles and their effect on the mechanisms of transportation of powdered materials 
in a laser cladding technique. The results of calculations presented below are obtained on the basis of mathematical models developed in Laboratory of Physics of Plasma Arc and Laser Processes (PPA\&LP Lab) of ITAM SB RAS using its own software, and ANSYS FLUENT and OpenFOAM codes [3,4].

\section{Lateral powder feed}

Off-axis powder injection into a laser spot is known from ancient times. Particle feeding was initially carried out through a simple tube, later - through a double cylindrical nozzle with the organization of the annular flow of protective gas from the oxidation of the ambient air. Off-axis powder injection became known as lateral injection. The position of injector is determined by the angle between nozzle and work piece and the distance between tip and work piece. Typical dimensions of one track of such a nozzle: width 0.5 $3.0 \mathrm{~mm}$; height $0,3-1,5 \mathrm{~mm}$. The design of the modern lateral nozzle uses a cyclone, Fig. 1 (a). This device is known as a separator for cleaning dusty gases [5]. It turned out that with the use of a cyclone it is possible to organize a more uniform supply of powder particles, eliminating the unstable flow regimes associated with fluctuations of the carrier gas.

The stability of the lateral nozzle is determined by the features of the flows within the cyclone, which is located in the upper part of the eccentric, Fig. 1 (a). The cyclone is a chamber with two tubes, one for injecting the powder, and the other for transporting it to the outlet nozzle-nozzle. The cyclone still has a hole with a diameter controlled by a special cap through which excess gas is discharged. The ratio of the flow rates of the transporting and bleeding gases is regulated by changing the diameter of the hole by installing the caps. The length of the laminar portion of the jet flowing out of the lateral nozzle is determined by the ratio of the flow rate of the supplied gas to the flow rate of the gas bleeding through the hole in the cap. When a gas-dispersed flow is inputted into the cyclone, a vortex gas flow is formed in which the powder particles are also involved. An increase in the concentration of particles on the cyclone wall and at the outlet from it is observed, Fig. 2 (b). At the same time, an inverse current appears on the axis of the cyclone, directed toward the hole in the cap, where an excess gas escapes, Fig. 2 (a).

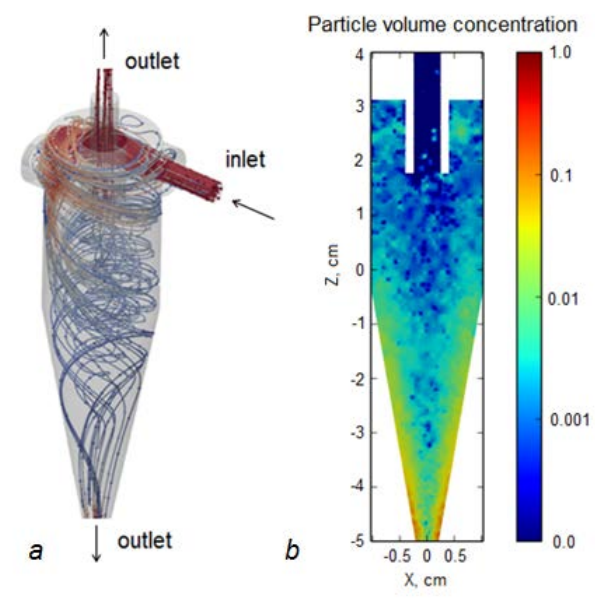

Fig. 2. Current stream-lines in the cyclone (a); and the concentration distribution of the powder (b). The calculation was carried out in ITAM SB RAS (PPA\&LP Lab) with the participation of Mr. A.M. Gurin. 
Despite the fact that the lateral nozzle does not provide for the focusing of the powder in the beam spot, it is convenient to use it to restore rotationally symmetric components of the items or for areas of processing that is difficult to access (grooves). With a lateral nozzle, high material deposition rates of up to $6 \mathrm{~kg} / \mathrm{h}$ are achieved. The lateral nozzle is used where it is required to ensure the duration of the process, i.e. for cladding for several hours.

\section{Multi-jet coaxial nozzle}

In laser cladding, a combination of the direction of powder transport with the direction of action of the laser beam is provided. In this case, the divided into several channels (into three or four ports) are distinguished and continuous (annular) focusing of the powder. Focusing of gas-powder jets from several separated channels is ensures the symmetry of the powder input relative to the direction of motion of the laser cladding head.

At the outlet from the separated channels, the powder particles can have a velocity of up to $15-20 \mathrm{~m} / \mathrm{s}$. Therefore, the multi-jet nozzle is less sensitive to a change in the direction of displacement relative to the plane of the substrate, and allows the head to be inclined in a wide range from the vertical. This allows processing large items without their additional movement in space. Multi-jet nozzles are used for simultaneous feeding of different types of powders, and the required distribution and concentration of particles in the depositing layer can be achieved. The choice of the multi-jet powder injection system ensures the generation of the focusing of particles with a diameter of the interaction zone of gaspowder jets of 1-3 mm. To clad the thicker layers, a laser with a power of 2 to $5 \mathrm{~kW}$ is used. It is possible to layer-by-layer depositing of three-dimensional items with overlapping or overhead contours. Figure 3 shows the change of powder density in the converging jet streams of the four-jet coaxial nozzle. Flow rates of gases: the carrier - $2.51 / \mathrm{min}$; protective - $10 \mathrm{l} / \mathrm{min}$, powder flow rate (stainless steel) $100 \mathrm{~g} / \mathrm{min}$. A four-jet head with a channel angle of $25^{\circ}$ focuses the powder micro jets with an axially symmetric particle density distribution at a distance of $25 \mathrm{~mm}$ from the output.
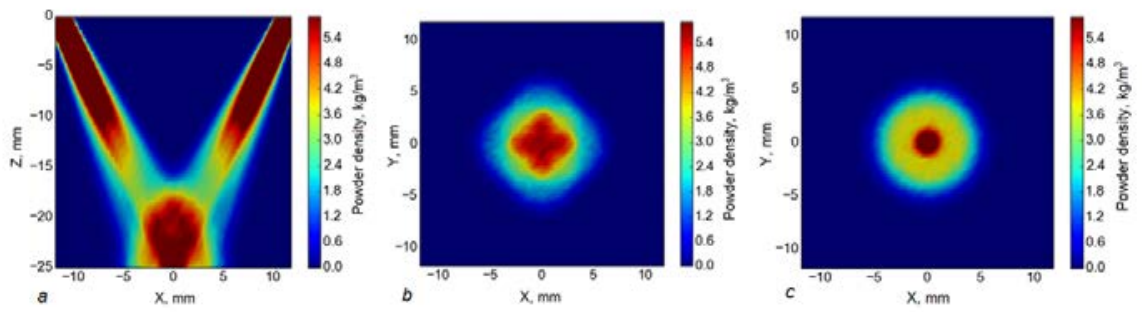

Fig. 3. Distribution of powder density in a gas-dispersed flow created by a four-jet coaxial nozzle. Plane $O x z$, parallel to the beam ( $a$ ); plane $O x y$, perpendicular to the beam: for $z=20 \mathrm{~mm}(b)$; and for $z=25 \mathrm{~mm}(c)$. The calculation was carried out in ITAM SB RAS (PPA\&LP Lab) with the participation of Mr. D.V. Bedenko.

Multi-jet focusing of powder flows is least likely to be affected by fluctuations in gas parameters and changes in the mass flow rate of the powder. Optimal technological parameters for the use of multi-jet nozzles can be established. Multi-jet nozzles are more economical and provide opportunities to improve the uniformity of the deposited layer. One of the advantages of these nozzles is that they can be used for processing by lasers that generate a laser beam with a different cross-section - circular or rectangular. 


\section{Annular coaxial nozzle}

The continuous supply of the powder differs from the divided (multi-jet) supply in that the particles are distributed in a conical annular channel, forming a powdery (gas + particle) cone that surrounds the laser beam [6]. The internal arrangement of the annular coaxial nozzle consists of concentric (nested into each other) cones with adjustable gaps between them. These gaps form annular channels through which powder, protective, carrier and forming gases are fed. The value of annular cross-sections is established due to displacement along the axis of internal cones, which are made mobile. The nozzles differ in the angle of narrowing of the cones, which ensures a completely defined angle of entry of particles into the laser beam zone [7]. This makes it possible to reduce the size of the focusing area of the powder flow to $0.2 \mathrm{~mm}$, and to apply laser surfacing with high accuracy and efficiency even with a small diameter of the laser beam [8]. The localization of the powder jet, the degree of heating and melting of particles in the laser light field are responsible for the quality and properties of the items obtained. For powdered compositions capable to thermal decomposition, such as tungsten carbide (WC), for example, heating and melting particles in the laser light field before they enter the molten pool is highly undesirable. The internal structure of the powder jet and its dynamics is influenced by the geometry of the nozzle, the particle size distribution and the physical properties of the powder material. At low powder mass flux, it is possible to obtain an ideal focusing of gasdispersed flows, which is disturbed as the mass flux increases. In some cases, the depositing conditions can be controlled by increasing the laser power and the mutual location of the beam caustic, the powder focusing area, and the position of the substrate plane to maintain the deposition efficiency and minimize the cladding defects [8].

Software implementations of models are partially verified by the results of experiments on visualization of particle dynamics. However, it turned out that these models work well only at low powder mass flux. As a result of the conducted studies, it was shown that the dimensions of the annular channels, the geometry of the converging and outlet sections of the nozzle affect the parameters of the scattering of the gas-dispersed jet [7,8]. With a constant flowrates of gas and powder, the distribution of particles in the powder jet is constant and depends on the particle size and shape, density of the material, and channel geometry. The collisions of particles with the nozzle walls affect the trajectories of the particles, the geometry of the profile of the powder jet, and the density of the particle distribution in the flow and on the substrate.

The particles of spherical shape are well entrained in gas and are better transported in narrow channels by laminar flow. Particles of irregular shape move with greater chaos, as a result they are poorly focused, and give a high scattering angle of the powder jet [7]. Inelastic particle collisions with the walls are the cause of the decrease in the velocity after reflection, which positively affects on the powder jet profile and focusing. The heating of the particles by laser depends on the position of their trajectories and the residence time in the region of high radiation intensity. Since the shape of the particles depends on the technology of their production, the results of the computational and experimental studies can also be used to formulate the requirements for the method for obtaining powders. With an increase of powder flowrate in the outflowing stream pulsations are appear, and powder jet becomes unsteady and weakly controlled. It was found in the calculations that the absolutely elastic or inelastic interaction mechanism embedded in the model of reflection of particles from the nozzle wall has a significant effect on the shape and focusing of the powder jet.

The geometry of the nozzle Trumpf, Fig. 4, has a focusing of the powder, which depends on the chosen law of reflection of particles from the nozzle wall (absolutely elastic or inelastic) [7]. The influence of the law of particle reflection, the position of their 
trajectory space and the overall profile of the powder jet are shown in Fig. 4, in the case of absolutely elastic (a), and inelastic (d) reflection laws. Changes in the distribution of the density of the powder in the gas-dispersed flow in the $O x z$ plane are shown in Fig. 4 (b, e). In Fig. 4 (c, f) shows changes in the mass flow of particles on the substrate in the Oxy plane. The distribution of the mass flow of the powder in the plane of the substrate depends on its position relative to the nozzle [7].

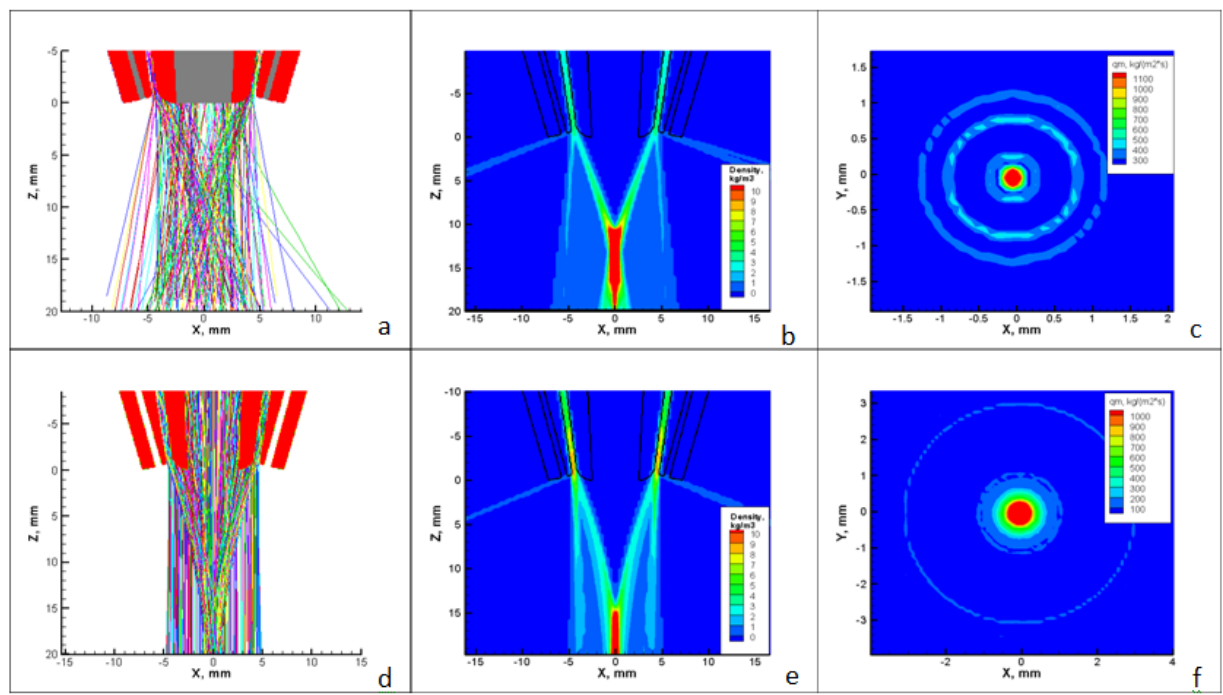

Fig.4. Calculation of the annular coaxial nozzle and powder jet parameters: particle trajectories (a, d); powder density in the axial section $O x z$ (b, e); distribution of mass particle flow on the substrate (c, f).

The author gratefully appreciates the financial support from the Russian Scientific Fund (contract No. 18-19-00430).

\section{References}

1. E. Toyserkani, A. Khajepour, S. Corbin, Laser Cladding (CRC PRESS, Boca Raton, London, New York, Washington, D.C. 2005)

2. S. Lester, N. Longfield, J. Griffiths, J. Cocker, C. Staudenmaier, G. Broadhead, New systems for laser cladding, laser surface modifications in steel industry, Laser Technik J., v. 10, 3, pp.41-43 (2013)

3. O.B. Kovalev, A.V. Zaitsev, D. Novichenko, I. Smurov, Theoretical and experimental investigation of gas flows, powder transport and heating in coaxial laser direct metal deposition (DMD) process, J. Therm. Spray Technol., v. 20, pp. 465-478 (2011)

4. O.B. Kovalev, Modeling of processes in laser additive manufacturing of $3 D$ metal items, Bull. Russian Acad. Scien. Phys., v. 80, 4, pp. 367-372 (2016)

5. A. Raoufi, M. Shams, M. Farzaneh, R. Ebrahimi, Numerical simulation and optimization of fluid flow in cyclone vortex finder, Chem. Engin. Proc., v. 47, pp. 128137 (2008)

6. D.V. Bedenko, O.B. Kovalev, I. Smurov, A.V. Zaitsev, Numerical simulation of transport phenomena, formation the bead and thermal behavior in application to industrial DMD technology, Int. J. Heat and Mass Transf., v. 95, pp. 902-912 (2016) 
7. O.B. Kovalev, I.O. Kovaleva, I. Yu. Smurov, Numerical investigation of gas-disperse jet flows created by coaxial nozzles during the laser direct material deposition, J. Mater. Proces. Tech., v. 249, pp. 118-127 (2017)

8. O.B. Kovalev, D.V. Bedenko, A.V. Zaitsev, Development and application of laser cladding modeling technique: From coaxial powder feeding to surface deposition and bead formation, Appl. Math. Model., v. 57, pp. 339-359 (2018) 